\title{
Comparison of microRNA expression in hippocampus and the marginal division (MrD) of the neostriatum in rats
}

\author{
Si Yun Shu ${ }^{1 *}$, Di Qing ${ }^{1}$, Bin Wang ${ }^{1 *}$, Qi-yi Zeng ${ }^{1}$, Yan-chen Chen ${ }^{1}$, Ying Jin ${ }^{2}$, Chang-chun Zeng ${ }^{2}$ and Rong Bao ${ }^{3}$
}

\begin{abstract}
Background: MicroRNAs (miRNAs), a class of highly conserved small non-coding RNA molecules, are known to play essential roles in central nervous system (CNS) by causing post-transcriptional gene silencing. There is much evidence that miRNAs have specific temporal and spatial expression patterns in the mammal brain, but little is known about the role of the region specificity for the gene regulatory networks of the brain. This study represents the first attempt to perform a profiling analysis of the differential expression of miRNAs between hippocampus and the Marginal division (MrD) of the neostriatum in the rat brain.
\end{abstract}

Results: Microarray was used to detect the expression of 357 miRNAs in hippocampus and the MrD from three rats. A short-list of the most dysregulated 30 miRNAs per rat was generated for data analysis, and the miRNAs that were represented in two or three short-lists were then further analyzed. Quantitative real-time reverse transcriptionpolymerase chain reaction (RT-PCR) was employed to validate the aberrantly expressed miRNAs obtained from the miRNA microarray analysis. A family of 11 miRNAs demonstrated differential expression between the MrD and hippocampus in more than one rat. Amongst these, miR-383 was differentially expressed in all three rats and upregulated to the largest degree in rat one, and the ten other miRNAs, let-7d*, miR-181b, miR-187, miR-195, miR-214, miR-382, miR-411, miR-466b, miR-592 and miR-1224 were differentially expressed in at least two rats. Of these ten, besides miR-382 and miR-411 which were up-regulated in one rat and down-regulated in another, the other eight miRNAs retained a uniform direction of regulation (up-regulation or down-regulation) between different specimens. When further examined by RT-PCR, the aberrantly expressed miRNAs, except miR-383 and let-7d*, demonstrated differential expression that significantly correlated with the microarray findings.

Conclusion: This study reported that the miRNA expression patterns in MrD was distinct from that of Hip, suggesting the role of miRNAs in the learning and memory function of the MrD probably different from hippocampus.

Keywords: mRNA transcript, Oligo-microarray, Gene silence, Synapse formation, Learning and memory

\section{Background}

In 1993, Lee etc. firstly found miRNAs in the nematode C. elegans to be key regulators of developmental transitions [1], and since then miRNAs have been identified in species ranging from plants to humans [2,3]. It has been shown that miRNAs regulate expression of $30 \%$ or more of animal genes [4]. The first form of MiRNAs were long primary transcripts (pri-miRNAs),

\footnotetext{
*Correspondence: shusybao@gmail.com; wangbin6556@126.com ${ }^{1}$ Center of Pediatrics, Zhujiang Hospital, Southern Medical University, Guangzhou, Guangdong 510280, China

Full list of author information is available at the end of the article
}

which are spilt into approximately $70 \mathrm{nt}$ stem-loop precursors (pre-miRNAs) and then further processed to mature miRNAs in the cytoplasm by the RNaseIII Dicer [5]. MiRNAs, which are approximately 19-23 nucleotides in length, are post-transcriptional regulators that bind to complementary sequences in $3^{\prime}$-UTRs (Untranslated regions) of target mRNA transcripts, usually resulting in gene silencing [6,7]. Upon miRNA binding, mRNAs are then localized to the processing bodies (P-bodies), where they are either deadenylated and degraded or translationally inhibited [8]. The extent of complementarity between miRNAs and their targets 
may influence whether transcripts are degraded [3]. MiRNAs are expressed in a number of cell types at differing levels, and are especially abundant in the central nervous system, suggesting that they might be particularly important there [9].

The function of miRNAs in nervous system development has been identified initially through classical forward genetic approaches [10]. Recent evidence points to a widespread role for neural miRNAs at various stages of synaptic development, including dendritogenesis, synapse formation and synapse maturation [11]. It is well known that synapses mediate communication between nerve cells, and contribute to learning and memory. Besides synaptic function, the proposed roles of miRNAs in the vertebrate CNS may include neurogenesis [12], regulation of morphogenesis [13], dendrite formation [14], and silencing of non-neural mRNAs [15]. In another paper, miRNAs were shown to be involved in memory [16]. Furthermore, a number of studies indicated that miRNAs might contribute to the control of synapse function and plasticity in the adult [17]. All of these functions of miRNAs indicated that it plays an important role in mediating regulation of mRNA expression and function by changes in neuronal activity. Dendritogenesis, synapse formation and new protein synthesis have long been recognized critical for formation of long-term memories (LTMs) [18]. Additionally, miRNAs were found as biomarkers for cancer and other disorders [19], and are involved in the etiology of several brain disorders, including Parkinson's disease [20], Alzheimer's disease [21], and depression [22].

The MrD is a pan-shaped subdivision in the caudal border of the neostriatum surrounding the rostral edge of the globus pallidus [23]. The MrD was first discovered in the brains of rat, and then it was verified that it is a universal structure in the neostriatum of the mammalian rain, including rat, cat, monkey and humans [24]. The $\mathrm{MrD}$ consists of spindle-shaped neurons, with high level expression of certain neuropeptides, monoamines and their receptors, such as substance $\mathrm{P}$, dynorphin $\mathrm{B}$, neurokinin 1 receptor in the fibers, terminals and neuronal somata in the $\mathrm{MrD}$ by immunohistochemical and patch clamp methods [25-27]. The MrD was found to be involved in learning and memory by double-blind studies of Y-maze learning and long-term potentiation in rats [28]. The MrD is a new component of the limbic system and is a key linking region between the limbic system and the basal nucleus of Meynert. Functional magnetic resonance image (fMRI) studies illustrated that the MrD and the prefrontal cortex are involved in digital working memory in the human brain [29]. The MrD has been shown to contribute to associative learning and declarative memory by behavioral study in rats and by fMRI study in humans. Lesions in the $\mathrm{MrD}$ influenced the learning and memory function of the basal nucleus of Meynert and attenuated hippocampal long-term potentiation [30].

The hippocampus is a well established learning and memory-related area of brain, that by comparison to the $\mathrm{MrD}$, has distinct biological functions. However some associations between the hip and the $\mathrm{MrD}$ are also found in many papers. Although the functional consequences of region-special expression of miRNAs is not yet known, we believe that the region-specific expression pattern may contribute to functional differences between the brain regions. The purpose of the current work is to investigate the miRNA expression in the $\mathrm{MrD}$ by comparison to that of in hippocampus by using microarray technology. The results of this study may be valuable to explain the mechanisms of learning and memory function of the MrD, and regulatory neural networks and will enable novel functional genomic analyses in the rat.

\section{Methods}

\section{Animals and total RNA extraction}

Adult male Sprague-Dawley rats $(\mathrm{N}=3)$, weighing about $220 \mathrm{~g}$, were used in this study. Food and water was available ad libitum, and rats were kept on a 12-h lightdark schedule (light period $6 \mathrm{am}-6 \mathrm{pm}$ ). The rats were deeply anesthetized with $10 \%$ chloral hydrate $(3.5 \mathrm{ml} / \mathrm{kg}$, i.p.) and then perfused, via the aorta, with $0.9 \%$ saline solution followed by cold $4 \%$ paraformaldehyde. The brains were removed in $4{ }^{\circ} \mathrm{C} 0.9 \%$ saline solution. The tissue samples of Hip and MrD in brain were cut about $1 \mathrm{~mm}^{3}$ respectively (Table 1). Total RNA populations were extracted from brain tissue using the miRNeasy Mini Kit (Qiagen GmbH, Hilden, Germany) and were subjected to undergone a quality analysis to determine the quality and the quantity of the sample RNA (Table 1). The quality control was carried out with the Agilent 2100 Bioalyzer, using the RNA 6000 Nano Kit according the manufacturer's recommendations. Within the resulting electropherogram, high quality RNA were characterized by two distinct bands, representing the 18 and $28 \mathrm{~s}$ rRNA (Figure 1:The electrophoretic patterns of RNA samples). All the samples were stored at $-70^{\circ} \mathrm{C}$ for detecting miRNA expression profile.

Table 1 The information of samples and RNA quality check

\begin{tabular}{lllll}
\hline Sample & Description & Source & OD260/280 & RIN* \\
\hline LZ1 & Hip 1 & hippocampus (Rat 1) & 1.99 & 8.6 \\
LZ2 & MrD 1 & Marginal division (Rat 1) & 1.99 & 7.8 \\
LZ3 & Hip 2 & hippocampus (Rat 2) & 2.08 & 8.7 \\
LZ4 & MrD 2 & Marginal division (Rat 2) & 1.93 & 8.4 \\
LZ5 & Hip 3 & hippocampus (Rat 3) & 2.02 & 8.8 \\
LZ6 & MrD 3 & Marginal division (Rat3) & 1.96 & 8.4 \\
\hline
\end{tabular}

*RIN: RNA integrity number. 


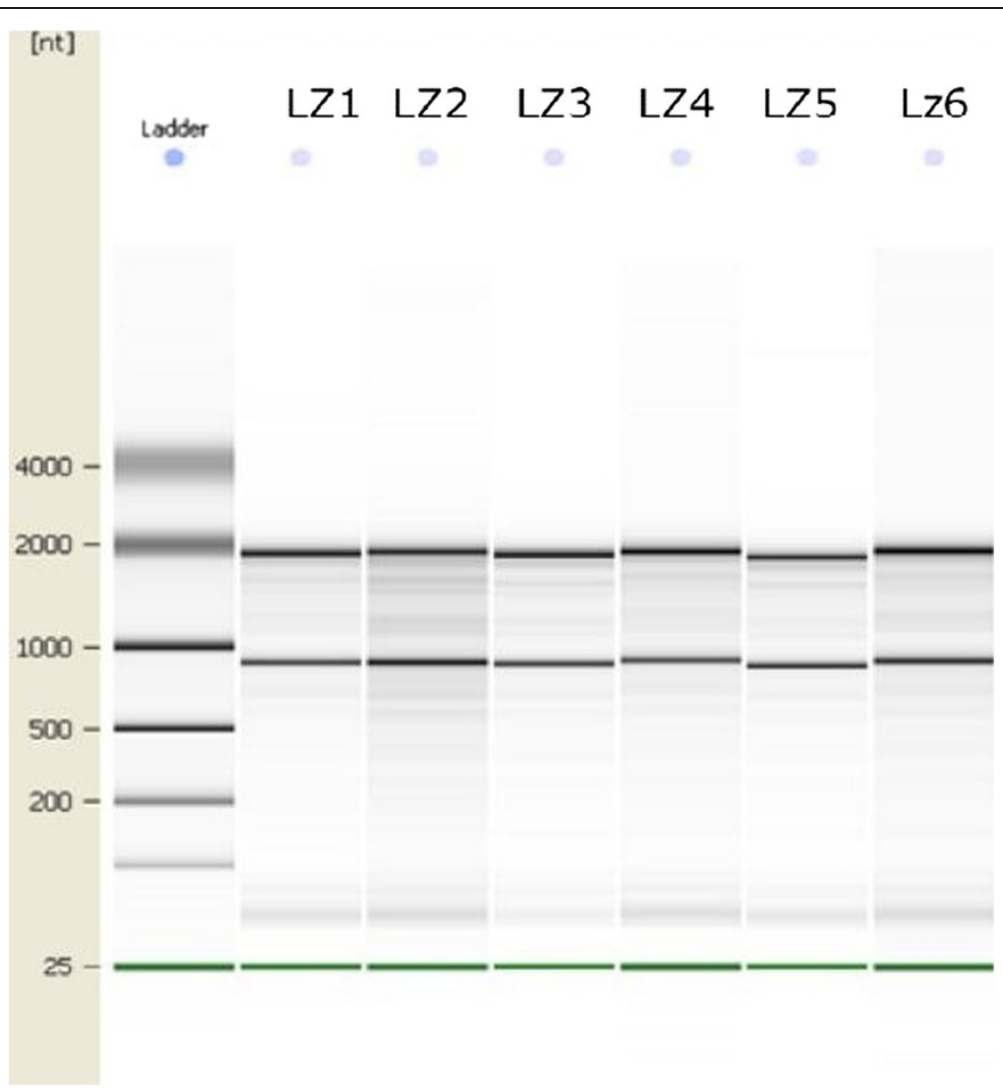

Figure 1 The electropherogram of the samples.

All studies conducted on animals were approved by the Institutional Animal Care and Use Committee(Southern medical university, Guangzhou,P.R. China).

\section{miRNA microarray screening}

Febits biochip "Geniom Biochip MPEA rattus norvegicus" was used for this analysis. These probes are designed as the reverse complements of all major mature miRNAs and the mature sequences as published in the current Sanger miRBase release (version 14.0 September 2009, see http://microran.sanger.ac.uk/seqences/index.shtml) [31] for rattus norvegicus.

Additional nucleotides are bound on the 5 'end of each capture oligonucleotide necessary for the enzymatic extention in the labeling procedure. The probes are synthesized with intra-array replicates to increase the statistical confidence and to compensate for potential positional effects. As a result the raw data files contain a total of 7 data points for each miRNA. The intensities of blank probes which consist only of one single " $T$ " nucleotide are used for background corrections. Spike-in controls for the labeling efficiency are also present. In order to correctly control the hybridization process as well as positioning features, additional hybridization controls are added to the array template. Blank, labeling control and hybridization control probes are not included in the data analysis.

The light-activated in-situ oligonucleotide synthesis using a digital micromirror device was performed within the Geniom One instrument on an activated threedimensional reaction carrier consisting of a glass-silicon -glass sandwich. Using standard DNA synthesis reagents and $3^{\prime}$-phosphoramidites carrying a $5^{\prime}$-photolabile protective group, oligonucleotides were synthesized in parallel in eight individual microchannels of one biochip. Prior to synthesis, the glass surface was activated by coating with a spacer to facilitate probe-target interaction and to avoid probe-probe interference [32].

For each array the RNA was suspended in Febit's proprietary miRNA Hybridization Buffer (25ul per array). Hybridization was carried out automatically for $16 \mathrm{~h}$ at $42^{\circ} \mathrm{C}$ using the Geniom RT-Analyzer. In the next step the biochip was given a stringent wash. Following the labeling procedure, the microfluidic-based primer extension assay was applied [33]. This assay utilizes the bound miRNAs as a primer for an enzymatic elongation with labeled nucleotides. The elongation was carried out with Klenow Fragment and biotinylated nucleotides at $37^{\circ} \mathrm{C}$ for 15 minutes. Finally, the biochip was washed automatically. 
For maximum sensitivity, biotin was used and detected with streptavidin-phycoerythrin (SAPE), in combination with febit's consecutive Signal Enhancement (CSE) procedure (Febit). For a more detailed description please the read febit protocol 010 from Febit (reference). The feature recognition (using Cy3 filter set) and signal calculation were done automatically within milliseconds. The Geniom Technology showed accurate detection of miRNA profiles. The data correlated well with qPCR data. There was no photo bleaching which enabled repeated measurements and multiple detection of each Biochip.

\section{Bioinformatics analysis}

The bioinformatics analysis starts with a summary of the measured data. Thereafter, spatial effects on the chip are investigated and corrected. Then, the intensity value distribution of raw data is analyzed and normalized. To evaluate the distribution of signal on the biochips, we computed a spatial distribution plot. Such plots show the reactivity of each transcript of the 8 arrays at the current position of each transcript. To account for variation between the hybridized arrays normalization is essential. For miRNAs, the VSN (variance stabilizing normalization) may outperform other approaches such as the quantile normalization. Applying quantile normalization, it is assumed that all arrays should show exactly the same distribution. However, especially in the case of miRNA, the underlying assumptions may not hold. In contrast, VSN is derived from a model of the variance-versus-mean dependence. While the variance of transcripts should be independent of their mean, measured data often show a quadratic dependency. This quadratic dependency can also be detected in the measured data.

Higher-level bioinformatics analyses, including correlation analysis, scatter plots, cluster analysis, venn diagrams, MA plots, principal component analysis, variance-related boxplots and so on, are executed. These analyses may help enhance our understanding to understand the principles of the applied approaches and may facilitate the interpretation of the results.

\section{RT-PCR}

The RNA were extracted from 5 adult male SpragueDawley rats, we got the tissue samples of Hip and MrD in brain using the same way showed as above. A quantitative PCR was performed using a miScript SYBR-Green PCR Kit (Qiagen) following the manufacturer's protocol. All qRT-PCR assays were done in triplicate for each sample. Briefly, for each miRNA to be assayed, $20 \mathrm{ng}$ of total RNA was converted to cDNA using the miRNAspecific Taqman primer. The resulting cDNA was diluted 1:2 prior to performing qRT-PCR. The small nuclear RNA U6 was used as the normalization control.
The 11 aberrantly expressed miRNA expression level was calculated with the CT method using the ABI 7300 Sequence Detection System (Applied Biosystems, Foster City, CA, USA).

\section{Statistical analysis}

Results are presented as the mean \pm standard deviation. Statistical analyses were carried out using spss13.0 software. The comparison of miRNA expression examed by RT-PCR between hippocampus and the Marginal division $(\mathrm{MrD})$ was carried out using independent t-test. $\mathrm{P}<0.05$ was considered statistically significant.

\section{Results}

\section{miRNA array data}

The miRNA expression profiles derived from different brain tissues were analyzed using oligo-microarray. For the detection of differentially regulated miRNAs, we focused on the following comparisons: LZ1 vs. LZ2, LZ3 vs. LZ4 and LZ5 vs. LZ6. Properties of the raw data are presented in Table 2. As the tables show, we have only about $0.04 \%$ flagged values. The mean background varied between the Biochips indicating that a background correction of the data is appropriate (details on the background correction are not shown).

\section{miRNA differential expression between Hip and MrD}

To investigate the differential expression of miRNAs, array-based miRNA profiling of rat Hip and the MrD was performed. In each specimen (rat1, rat2 and rat3), we generated a short-list of the miRNAs showing greatest up- or down-regulation. The following table shows the 30 probes with the largest changes in expression (detected by highest absolute value of log fold changes) for the above comparisons (Additional file 1: Table S3).

In these most deregulated expression of miRNAs, we identified eleven miRNAs which occurred high differential expression in more than one rat simultaneously (gray lines in Additional file 1: Table S3). Hence, miR-383 was differentially expressed in three rats, and let-7d*, miR-181b, miR-187, miR-195, miR-214, miR-382, miR-411, miR-

Table 2 Summary on raw data of miRNA array

\begin{tabular}{lcccccc}
\hline & LZ1 & LZ2 & LZ3 & LZ4 & LZ5 & LZ6 \\
\hline Mean intensity & 720 & 612 & 530 & 749 & 599 & 1168 \\
sd intensity & 1572 & 1398 & 811 & 759 & 1085 & 3182 \\
Mean bg. int. & 256 & 240 & 288 & 512 & 352 & 248 \\
sd bg. int. & 57 & 40 & 69 & 117 & 74 & 54 \\
Present calls & 2462 & 2380 & 1345 & 856 & 1036 & 3395 \\
Present calls\% & 42 & 40 & 23 & 14 & 17 & 58 \\
Flagged & 88 & 130 & 322 & 63 & 180 & 14 \\
\hline
\end{tabular}


466b, miR-592, miR-1224 were differentially expressed in two rats at least (Additional file 2: Table S4). Beside miR382 and miR-411 which were up-regulated in one rat and down-regulated in another, the other nine miRNAs kept the uniform regulated direction (up or down) between different rats (Additional file 2: Table S4).

\section{RT-PCR}

To validate the miRNA microarray results, 11 differently expressed miRNAs was analyzed by real-time RT-PCR. Every miRNA has $3 \mathrm{CT}$ values, the mean $\mathrm{CT}$ values were showed in the Table 3. We found that except miR-383 and let-7 $\mathrm{d}^{*}$, the other 9 miRNAs were significantly higher expressed in the MrD. The RT-PCR results were partly in concordance with the miRNA microarray analysis results.

\section{Discussion}

The MrD of the neostriatum is a flat, pan-shaped structure consisting of dorsoventral spindle-shaped neurons arranged in a parallel formation, located at the caudomedial margin of the neostriatum and surrounding the dostrolateral border of the globus pallidus (GP), and distinct from other parts of the striatum in the mammalian brains. Lesion and Y-maze tests and assessment of c-Fos expression and Patch clamp analysis showed that the $\mathrm{MrD}$ contributes to the learning and memory function[23-30]. FMRI investigations and clinical case reports verified the contribution of the $\mathrm{MrD}$ to digital working and mathematical calculating memory in the human brain[34-36]. All these early experiments indicate that $\mathrm{MrD}$ is likely to be an important subcortical center of learning and memory based on its position, its advanced development in higher mammalian brains, its abundant blood supply and the diverse connections with other memory-related structures. Moreover, miRNAs are

Table 3 The results of RT-PCR of 11 aberrantly expressed miRNAs

\begin{tabular}{lccc}
\hline & $\begin{array}{c}\text { The mean CT } \\
\text { value of Hip }\end{array}$ & $\begin{array}{l}\text { The mean CT } \\
\text { value of MrD }\end{array}$ & p-value \\
\hline rno-let-7d* & $19.16 \pm 0.05$ & $19.23 \pm 0.14$ & 0.63 \\
rno-miR-181b & $16.68 \pm 0.05$ & $17.62 \pm 0.19$ & 0.00 \\
rno-miR-187 & $25.31 \pm 0.08$ & $26.84 \pm 0.19$ & 0.00 \\
rno-miR-195 & $23.11 \pm 0.08$ & $24.34 \pm 0.14$ & 0.00 \\
rno-miR-214 & $22.47 \pm 0.10$ & $23.49 \pm 0.20$ & 0.00 \\
rno-miR-382 & $24.88 \pm 0.18$ & $26.04 \pm 0.17$ & 0.00 \\
rno-miR-383 & $24.06 \pm 0.03$ & $24.36 \pm 0.14$ & 0.06 \\
rno-miR-411 & $23.94 \pm 0.21$ & $26.39 \pm 0.33$ & 0.00 \\
rno-miR-466b & $18.96 \pm 0.08$ & $19.50 \pm 0.08$ & 0.00 \\
rno-miR-592 & $24.38 \pm 0.10$ & $25.45 \pm 0.08$ & 0.00 \\
rno-miR-1224 & $21.97 \pm 0.21$ & $22.94 \pm 0.24$ & 0.01 \\
\hline
\end{tabular}

particularly abundant in neurons, and together with the fact that a given miRNA usually regulates the expression of hundreds of target mRNAs, neuronal miRNA pathways create an extremely powerful mechanism for dynamically adjusting the protein content of neuronal compartments without the need for new gene transcription. MiRNAs likely have a big impact on higher cognitive function including learning and memory [10,37,38]. It has been speculated that miRNA expression differing from brain region to region, may reflect brain region-specific miRNA expression patterns with a corresponding role in each brain area [39]. Recently, Juhila et al. compared miRNA expression between hippocampus and frontal cortex, and confirmed this hypothesis finding that miRNA expression in the hippocampus was extremely different from that of frontal cortex [40]. Based on the above discoveries, this research was aimed at finding those miRNAs that were differentially expressed between $\mathrm{MrD}$ and Hip, the well established memory related structures in the brain.

Although miRNAs have been implicated in several important biological functions in the CNS including neurogenesis [41], dendrite formation [42], brain morphogenesis [43], neural plasticity [44], and silencing of non-neuronal transcripts [45,46], far less information is available on miRNA expression patterns of neostriatum in anatomically regional differences. In this study, a miRNA array with 357 known rodent miRNA probes (15 replicates per array) was applied to sample the differential expression of miRNAs in Hip and the MrD of the rat brain. In the results, the most dysregulated 30 probes were identified in each rat. A total of 78 miRNAs were listed in Additional file 1: Table S3 and 11 miRNAs were differentially expressed in more than one rat (gray line in Additional file 1: Table S3). Although the most upregulated miRNAs in three rats were miR-383, miR451 and miR-219-5p (with logs [FC]: -2.15, -2.21, -2.60) respectively, we wanted to focus on miRNAs that were altered in different rats simultaneously. Notably, miR383 was not only most upregulated in rat1, but was also high in rat 2 and rat 3 . The other ten miRNAs, upregulated or down-regulated, were found to be high differentially affected in two different sample rats (Additional file 2: Table S4).

The importance of miRNAs in the nervous system was first described in Danio rerio (zebrafish) in which a mutation in dicer1 led to failure to produce mature miRNAs and resulted in gross morphological defects in the nervous system [47]. Effects involving regulation of specific miRNAs in neurons have been found in a number of organisms including mammals. For example, during neurogenesis, the levels of both miR-124 and miR-9 are greatly increased, and both of them were indicated involving in neuronal differentiation in vitro experiments $[44,48]$. Deep sequencing of miRNAs derived from tissues and cell lines have revealed these and 
other miRNAs are restricted to the CNS [46]. Definitive proof of the role of miR-124 in neurogenesis has now been achieved in vivo, revealing its critical role in the differentiation of neurons from neural precursors [49]. In addition to differentiation of neurons, miRNAs have been shown to affect crucial aspects of neurons. For example, neurite outgrowth is regulated by miR-132 [50]. Furthermore, one crucial functional aspect of neurons, the synapse, is under miRNAs control. In the hippocampus, miR-134 regulates the size of dendritic spines, sites of synaptic transmission [42]. Further linking of miRNAs to synaptic changes and the implications of such in brain development and plasticity was the recent demonstration that miR-138 controls dendritic spine morphogenesis [51]. The expression of miRNAs mentioned above was not significantly deregulated in our study, though they were verified to be more important in development, differentiation and function of CNS.

Rno-miR-383, the most interesting one of these miRNAs, is significantly up-regulated in Hip in all three rats. Its precursor possesses $73 \mathrm{nt}$ stem loop construction, and the mature sequence is $5^{\prime}$-cagaucagaaggugacugugg $-3^{\prime}$. After bioinformatics analysis, there are 770 predicted target sites in rattus norvegicu genome (http://mirnamap. mbc.nctu.edu.tw/index.php). There are 17 members from different species that have been sequenced in the mir-383 family, including: bta, cfa, eca, gga, hsa, mdo, mml, mmu, oan, ppy, ptr, rno, ssc, tgu, xtr, aca, sha (http://www.mirbase. org/cgi-bin/mirna_summary.pl?fam=MIPF0000137). Experimentally, Lian et al [52] have reported that the expression of hsa-miR-383 is altered in testicular itssues of human patients, and they consider that a potential target of miR-383 may be GADD45G. GADD45G can induce apoptosis and inhibit cell growth in response to stress shock. Abnormal expressions of these proteins may have a significant impact on male infertility [53,54]. However, no evidence demonstrates how rno-miR-383 is involved into complicated regulatory net-works in CNS, especially in $\mathrm{MrD}$.

Beside rno-miR-383, there are also 10 miRNAs differentially expressed in only two rats in this study. In these 10 deregulated miRNAs, seven (let-7 $\mathrm{d}^{*}$, miR-181b, miR187, miR-214, miR-466b, miR-592, miR-1224) are up-regulated in two rats, and one (miR-195) is downregulated. The last two investigated, miR-382 and miR411 , were up-regulated in one rat and down-regulated in another. It is possible to speculate that non tissuespecific expression may be a reason for this unexpected expression pattern of some miRNAs, and meanwhile they were also expressed in many different tissues in random manner. The other reasons may be the intrinsic variability of these miRNAs in this brain region or the effect correlate with some behavioral variability. Except for let-7d*, the RT-PCR results showed the same trend with that of microarray, which means that it validated the expression patterns we found in our microarray experiments.

Our previous study has shown that the MrD-NBMhippocampus circuit may play an important role in modulating learning and memory [23-30]. Tissue-specific miRNAs expression patterns as determined in this study can make important contributions to understanding regulatory expression networks. Although difficult for small RNAs, there is no substitute for in situ hybridization studies to follow up on various experimental findings in order to assess the regions and cell types responsible for distinct miRNA expression patterns. Meanwhile, differential expression of identified miRNAs can be then assessed in models of disease or in diseased tissue itself. In summary, the study on miRNAs, their mRNA targets, and resulting changes in protein products will continue to be an exciting field of research, leading to a greater understanding of the regulatory effects of the miRNA.

\section{Conclusion}

This is the first evidence for the comparison of miRNA expression profiles between hippocampus and the $\mathrm{MrD}$ of the striatum in rat brain. In dysregulated miRNAs, let-7d*, miR-181b, miR-187, miR-214, miR-383, miR466b, miR-592, miR-1224 are significantly overexpressed in $\mathrm{MrD}$ compared to hippocampus, and miR-195 is downexpressed. More study is required to clarify the precise contributions of miRNAs in the $\mathrm{MrD}$ and the hippocampal circuit.

\section{Additional files}

Additional file 1: Table S3. Differentially regulated miRNAs expression between Hip and MrD.

Additional file 2: Table S4. The details of different expression of 11

miRNAs mentioned above.

\section{Competing interests}

We declare that we have no competing interests.

\section{Authors' contributions}

SYS designed the research, directed and organized the experiments and drafted the manuscript; BW organized the experiments; DQ participated the microarray experiments and jointed in the revising of manuscript. YCC and RB carried out the RT-PCR, CCZ did the date analysis, QYZ and YJ provided valuable opinions for revising the manuscript. All authors read and approved the final manuscript.

\section{Acknowledgments}

This project was supported by NSFC 81271524. We thank Professor Jan-Yan Wu very much for his excellent edition on this manuscript. We thank Professor Xiao-Dan Jiang for her technical support.

\section{Author details}

${ }^{1}$ Center of Pediatrics, Zhujiang Hospital, Southern Medical University, Guangzhou, Guangdong 510280, China. ${ }^{2}$ The College of Biophotonics, South China Normal University, Guangzhou, Guangdong 510631, China. ${ }^{3}$ Department of Pediatrics, Sun Yet-San Hospital, Zhong-Shan University, Guangzhou 510120, China. 
Received: 6 November 2012 Accepted: 1 February 2013

Published: 20 February 2013

\section{References}

1. Lee RC, Feinbaum RL, Ambros V: The C. elegans heterochronic gene lin-4 encodes small RNAs with antisense complementarity to lin-14. Cell 1993, 75:843-854.

2. Reinhart BJ, Slack FJ, Basson M, et al: The 21-nucleotied let-7 RNA regulates developmental timing in Caenorhabditis elegans. Nature 2000, 403:901-906.

3. Bartel DP: MiRNAs: genomics, biogenesis, mechanism and function. Cell 2004, 116:281-297.

4. Miranda KC, Huynh T, Tay Y, Ang YS, Tam WL, Thomson AM, Lim B, Rigoutsos I: A pattern-based method for the identification of microRNA binding sites and their corresponding heteroduplexes. Cell 2006, 126:1203-1217.

5. Gabriele $\mathrm{S}$, Reuben $\mathrm{S}$, Gerhard S: microRNAs in neurons: manifold regulatory roles at the synapse. Curr Opin Genet Dev 2011, 21:491-497.

6. Bartel DP: MiRNAs: target recognition and regulatory functions. Cell 2009, 136(2):215-233.

7. Bak M, Silahtaroglu A, Moller M, Christensen M, Rath MF, Skryabin B, Tomerup N, Kauppinen S: MicroRNA expression in the adult mouse central nervous system. RNA 2008, 14:432-444.

8. Brengues M, Teixeira D, Parker R: Movement of eukaryotic mRNAs between polysomes and cytoplasmic processing bodies. Science 2005, 310:486-489.

9. Gao FB: Post-transcriptional control of neuronal development by miRNA networks. Trends Neurosci 2008, 31:20-26.

10. Kosik KS: The neuronal miRNA system. Nature Rev. Neurosci. 2006, 7:911-920.

11. Wayman GA, et al: An activity-regulated miRNA controls dendritic lasticity by down-regulating p250GAP. Proc. Natl. Acad. Sci. USA 2008, 105:9093-9098.

12. Visvanathan J, Lee $S$, Lee B, Lee JW, Lee SK: The microRNA miR-124 antagonizes the anti-neural REST/SCP1 pathway during embryonic CNS development. Genes Dev 2007, 21:744-749.

13. Giraldez AJ, Cinalli RM, Glasner ME, Enright AJ, Thomson JM, Baskerville S, Hammond SM, Bartel DP, Schier AF: MicroRNAs regulate brain morphogenesis in zebrafish. Science 2005, 308:833-838.

14. Schratt GM, Tuebing F, Nigh EA, Kane CG, Sabatini ME, Kiebler M, Greenberg ME: A brain-specific microRNA regulates dendritic spine development. Nature 2006, 439:283-289.

15. Conaco C, Otto S, Han JJ, Mandel G: Reciprocal actions of REST and a microRNA promote neuronal identity. Proc Natl Acad Sci USA 2006 103:2422-2427.

16. Bredy TW, Lin Q, Wei W, Baker-Andresen D, Mattick JS: MicroRNA regulation of neural plasticity and memory. Neurobiol Learn Mem 2011, 96:89-94.

17. Rajasethupathy $P$, et al: Characterization of small RNAs in Aplysia reveals a role for miR-124 in constraining synatic plasticity through CREB. Neuron 2009, 63:803-817.

18. Gold PE: Protein synthesis and memory (Introduction). Neruobiol. Learn. Mem 2008, 89:199-200.

19. De Smaele E, Ferretti E, Gulino A: MicroRNAs as biomarkers for CNS cancer and other disorders. Brain Res 2010, 1338:100-11.

20. Kim J, Inoue K, Ishii J, Vanti WB, Voronov SV, et al: A MicroRNA feedback circuit in midbrain dopamine neurons. Science 2007, 317:1220-1224.

21. Wang WX, Rajeev BW, Stromberg AJ, Ren N, Tang G, et al: The expression of microRNA miR-107 decreases early in Alzheimer's disease and may accelerate disease progression through regulation of beta-site amyloid precursor protein-cleaving enzyme 1. J Neurosci 2008, 28:1213-1223.

22. Saus E, Soria V, Escaramis G, Vivarelli F, Crespo JM, et al: Genetic variants and abnormal processing of pre-miR-182, a circadian clock modulator, in major depression patients with late insomnia. Hum Mol Genet 2010, 19:4017-4025.

23. Shu SY, Peterson GR: The 'marginal division': a new subdivision in the neostriatum of rat. J Chem Neuroanat 1988, 1:147-163.

24. Shu SY, McGinty JF, Peterson GM: High density zinc-containing and dynorphin B-and, substance P-immunoreactive terminals in the marginal division of the rat striatum. Brain Res Bulletin. 1990, 24:201-205.
25. Shu SY, Bao XM, et al: A new subdivision of mammalian neostriatum with functional implications to learning and memory. J Neurosci Res 1999, 58:242-253

26. Zeng JX, Shu SY, Bao XM, Zou F, Ji AM, Ye JF: Properties of acetylcholine receptor ion channels in the acutely dissociated neurons of the marginal division in the rat striatum. Neurochem Res 1999, 24:1573-1576.

27. Shu SY, Bao XM, et al: New component of the limbic system: marginal division of the Neostriatum that links the limbic system to the basal nucleus of meynert. J Neurosci Res 2003, 71:751-757.

28. Shu SY, Bao XM, et al: Hippocampal long-term potentiation attenuated by lesions in the marginal division of neostriatum. J. Neurochem. Res 2003, 28:749-753.

29. Shu SY, Wu YM, Bao XM, Wen ZB, Huang FH, Fu QZ, Ning Q: A new area with function of learning and memory in the human brain: immunohistochemical and functional MRI analysis. Mol Psychiatry 2002, 7(9):1018-1022.

30. Xue-mei L, Si Yun S, Chang-chun Z, Ye-feng C, Kui-hua Z, Chuan-xing W, Jian $F$ : The role of substance $P$ in the marginal division of the neostriatum in learning and memory is mediated through the neurokinin 1 receptor in rats. Neurochem Res 2011, 36(10):1896-902.

31. Griffiths-jones $S$, Saini $S$, et al: miRBase: tools for miRNA genomics. Nucleic Acids Res 2008, 36:D154-158.

32. Baum M, Bielau S, et al: Validation of a novel, fully integrated and flexible microarray benchtop facility for gene expression profiling. Nucleic Acids Res 2003, 31:e151.

33. Vorwerk K, Ganter Y, et al: Microfluidic-based enzymatic on-chip labeling of miRNAs. New Biotechnol. 2008, 25:142-149.

34. Shu SY, Wu YM, Bao XM, Leonard B: Interactions among memory-related centers in the brain. J Neurosci Res 2003, 71:609-616.

35. Shu SY: Marginal division of the neostraitum: a subcortical memory center. J Biomed Sci 2003, 10:14-29.

36. Shu SY, Song C, Wu Y, et al: Learning and memory deficits caused by a lesion in the medial area of the left putamen in the human brain. CNS Spectr 2009, 14(9):473-476.

37. Schratt G: Fine-tuning neural gene expression with miRNAs. Curr Opin Neurobiol 2009, 19:213-219.

38. Schratt G: miRNAs at the synapse. Nature Rev Neurosci 2009, 10:842-849.

39. Kapsimali M, Kloosterman WP, de Bruijn E, Rosa F, Plasterk RH, et al: MicroRNAs show a wide diversity of expression profiles in the developing and mature central nervous system. Genome Biol 2007, 8:R173.

40. Juuso J, Tessa S", Katherine I, Daniel N, Pekka E, Aleksi K, Eija K, Dario G, liris $\mathrm{H}$ : MicroRNA expression profiling reveals MiRNA families regulating specific biological pathways in mouse frontal cortex and hippocampus. PLoS One 2011, 6(6):e21495.

41. Visvanathan J, Lee $S$, Lee $B$, et al: The miRNA miR-124 antagonizes the anti-neural REST/SCP1 pathway during embryonic CNS development. Genes Dev 2007, 21:744-749.

42. Schratt GM, Tuebing F, Nigh EA, et al: A brain-specific miRNA regulates dendritic spine development. Nature 2006, 439:283-289.

43. Giraldez AJ, Cinalli RM, Glasner ME, et al: MiRNAs regulate brain morphogenesis in zebrafish. Science 2005, 308:833-838.

44. Griggs EM, Young EJ, Rumbaugh G, Miller CA: MicroRNA-182 regulates amygdala-dependent memory formation. J Neurosci 2013, 33(4):1734-40.

45. Conaco C, Otto S, Han JJ, et al: Reciprocal actions of REST and a miRNA promote neuronal identity. Proc. Natl. Acad. Sci. USA 2006, 103:2422-2427.

46. Vo N, Klein ME, Varlamova O, et al: A cAMP-response element binding protein-induced miRNA regulates neuronal morphogenesis. Proc Natl Acad Sci USA 2005, 102:16426-16431.

47. Wienholds E, Koudijs MJ, van Eeden FJ, et al: The miRNA producing enzyme Dicer1 is essential for zebrafish development. Nat Genet 2003, 35:217-218

48. Krichevsky AM, Sonntag KC, Isacson O: Specific miRNAs modulate embryonic stem cell-derived neurogenesis. Stem Cells 2006, 24:857-864.

49. Landgraf $P$, et al: A mammalian miRNA expression atlas based on small RNA library sequencing. Cell 2007, 129:1401-1414.

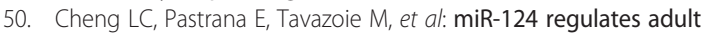
neurogenesis in the subventricular zone stem cell niche? Nat Neurosci 2009, 12:399-408.

51. Siegel $G$, et al: A functional screen implicates miRNA- 138-dependent regulation of the depalmitoylation enzyme APT1 in dendritic spine morphogenesis. Nat Cell Biol 2009, 11:705-716. 
52. Lian J, Zhang XS, Tian $\mathrm{H}$, et al: Altered miRNA expression in patients with non-obstructive azoospermia. BMC Reprod. Bio. Endocrinology 2009, 7:13.

53. Ying J, Srivastava G, Hsieh WS, et al: The stress-responsive gene GADD45G is a functional tumor suppressor, with its response to environmental stresses frequently disrupted epigenetically in multiple tumors. Clin Cancer Res 2005, 11:6442-6449.

54. Lian J, Tian H, Liu L, Zhang X-S, Li W-Q, Deng Y-M, Yao G-D, Yin M-M, Sun F: Downregulation of microRNA-383 is associated with male infertility and promotes testicular embryonal carcinoma cell proliferation by targeting IRF1. Cell Death Dis. 2010, 1:e94.

doi:10.1186/1423-0127-20-9

Cite this article as: Shu et al:: Comparison of microRNA expression in hippocampus and the marginal division (MrD) of the neostriatum in rats. Journal of Biomedical Science 2013 20:9.

\section{Submit your next manuscript to BioMed Central and take full advantage of:}

- Convenient online submission

- Thorough peer review

- No space constraints or color figure charges

- Immediate publication on acceptance

- Inclusion in PubMed, CAS, Scopus and Google Scholar

- Research which is freely available for redistribution 